\section{Negative Excess Enthalpy in a van der Waals Polymer Mixture}

\author{
D. W. Tomlin and C. M. Roland \\ Chemistry Division, Code 6120, Naval Research Laboratory, \\ Washington, D.C. 20375-5000
}

Received December 3, 1991

Revised Manuscript Received January 23, 1992

\section{Introduction}

Thermodynamic miscibility in high polymer mixtures is almost always the result of specific interactions between the components. However, there are a few blends which exhibit miscibility apparently without any specific interactions. ${ }^{1-4}$ If the like and unlike contact energies are nearly equivalent, miscibility can arise simply due to the combinatorial entropy. Poly(vinylethylene) (PVE) and cis-1,4-polyisoprene (PIP) are thermodynamically miscible as evidenced by glass transition measurements, ${ }^{5}$ proton and C-13 NMR, ${ }^{6}$ and the observation of spontaneous mixing of the components. 7,8 Infrared spectroscopy has demonstrated the absence of specific interactions between these essentially nonpolar hydrocarbons. ${ }^{9,10}$ Although it is expected that a van der Waals mixture (i.e., a blend characterized by only dispersion forces between the nonpolar components) will exhibit phase separation at sufficiently high component molecular weights, ${ }^{11}$ recently it was found that even cross-linked PIP forms miscible mixtures with high molecular weight PVE. ${ }^{12,13}$ Miscibility persists despite an almost negligible combinatorial entropy contribution to the free energy of mixing. If the common assumption is correct that van der Waals mixtures have an endothermic heat of mixing, ${ }^{14-17}$ this implies an astonishing equivalence in the van der Waals energy of the two polymers and no equation of state effects. ${ }^{11,17,18}$ However, substitution of deuterons for the protons of the PVE, which significantly alters the polarizability and hence the van der Waals energy of this polymer, ${ }^{19,20}$ does not induce phase separation in blends with PIP. 8 This result suggests the possibility that the interaction parameter, $\chi$, which provides a measure of all contributions to the mixing free energy other than the noncombinatorial entropy, may be negative for PIP/PVE mixtures, notwithstanding the absence of specific interactions. The SANS measurements described herein were carried out to examine this possibility.

\section{Experimental Section}

The protonated 1,4-polyisoprene, composed of $70 \%$ cis units, was obtained from Polymer Laboratories, Ltd. The perdeuterated poly(vinylethylene) was synthesized by C. A. Trask using tert-butyllithium as the initiator. The presence of 1,2-dipiperidinoethane and anisole during the reaction effected $97 \% 1,2$ addition of the butadiene monomer. ${ }^{21,22}$ As determined by gel permeation chromatography the degree of polymerization, $z$, equaled 5097 and 1691 for the PVE and PIP, respectively (respective polydispersities were 1.4 and 1.0). A blend containing a volume fraction $\phi=0.52$ of PIP was prepared by dissolving the polymers in hexane, with $0.05 \%$ by weight of a styrenated diphenylamine added as an antioxidant. A $2.5 \mathrm{~mm}$ thick film was cast and then dried in vacuum at $40^{\circ} \mathrm{C}$ for 1 day. The sample was sandwiched between two oxygen-free copper disks ( $0.2 \mathrm{~mm}$ thick) and mounted in a heating block attached to the SANS instrument.

The SANS measurements were carried out at the National Institute of Standards and Technology. ${ }^{23}$ Measurements were made at a series of temperatures, with the latter controlled to $\pm 0.6 \mathrm{~K}$. The monochromatic neutron beam had a wavelength $\lambda$ $=9.0 \AA$, and a $3.6-\mathrm{m}$ sample to detector distance was utilized. Circularly averaged intensities were obtained over a range of $q$
$(=4 \pi \sin \theta / \lambda$, where $2 \theta$ is the scattering angle) between 0.008 and $0.08 \AA^{-1}$. The scattering data were corrected for sample transmittance and electronic and background noise. Absolute intensities were determined using dry silica gel as a secondary standard.

\section{Results}

The SANS intensity from a single-phase polymer blend is composed of coherent scattering and an incoherent background:

$$
I(q)=S(q)+I_{\text {inc }}
$$

For an incompressible fluid (no density fluctuations) the random-phase approximation provides an expression for the coherent intensity arising from concentration fluctuations ${ }^{24,25}$

$$
k / S(q)=\left[z_{1} \phi_{1} v_{1} g_{1}(q)\right]^{-1}+\left[z_{2} \phi_{2} v_{2} g_{2}(q)\right]-\left(2 \chi / v_{0}\right)
$$

with

$$
k=N\left(\frac{b_{1}}{v_{1}}-\frac{b_{2}}{v_{2}}\right)^{2}
$$

In this expression $N$ is Avogadro's number and $b_{i}$ is the coherent scattering length for the $i$ th component. The lattice cell volume, $v_{0}$, is defined to be the geometric mean of the components' repeat unit molar volumes:

$$
v_{0}=\left(v^{1} v_{2}\right)^{1 / 2}
$$

The scattering function for a Gaussian coil depends only on the radius of gyration

$$
g_{i}(q)=2\left[R_{i}{ }^{2} q^{2}+\exp \left(-R_{i}{ }^{2} q^{2}\right)-1\right] / R_{i}{ }^{4} q^{4}
$$

which can be expressed in terms of the statistical segment length, $a_{i}$, of the polymer:

$$
R_{i}=a_{i}\left(z_{i} / 6\right)^{1 / 2}
$$

At small angles for which $q R_{i}<1$ eq 2 can be rewritten in the Ornstein-Zernike form ${ }^{24}$

$$
k / S(q)=a_{0}+a_{1} q^{2}
$$

whereby the zero-angle intercept of a plot of $S(q)^{-1}$ vs $q^{2}$ yields $\chi$ directly

$$
a_{0}=\left(2 / v_{0}\right)\left[\chi_{8}-\chi\right]
$$

The spinodal value of the interaction parameter is given by

$$
\chi_{\mathrm{s}}=\left(v_{0} / 2\right)\left[\frac{1}{z_{1} \phi_{1} v_{1}}+\frac{1}{z_{2} \phi_{2} v_{2}}\right]
$$

although this relation strictly applies only to "regular" mixtures, whose entropy change due to mixing is only com. binatorial; eq 8 also assumes an interaction parameter independent of composition. The resolution of the present experiments was insufficient to allow the use of the Ornstein-Zernike approximation. This is illustrated in Figure 1, where the linear extrapolation according to eq 6 is seen to overestimate the scattering intensity and hence would overestimate $\chi$.

Setting the quantity $k$ of eq 3 equal to $6.57 \times 10^{-3}$, the interaction parameter for the PVE/PIP blend was determined by fitting eqs 1 and 2 to the SANS data. Nonlinear regression was carried out by varying $\chi, I_{\mathrm{inc}}$, and an average statistical segment length defined as

$$
\mathbf{a}_{0}=\left(\mathbf{v}_{0} / \mathbf{v}_{i}\right)^{1 / 3} \mathbf{a}_{i}
$$

Alternatively, the statistical segment length reported for 


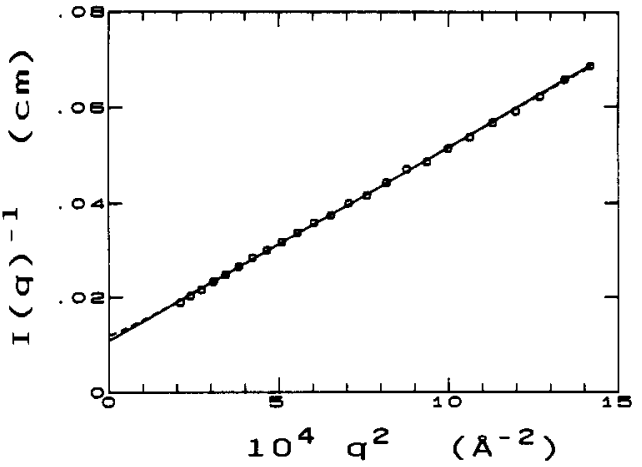

Figure 1. Scattered intensity measured for the blend at $345 \mathrm{~K}$ displayed in the Ornstein-Zernike form along with the best fit of eq $2(-\cdots)$. The data deviate from linearity (indicated by the solid line) at low $q$.

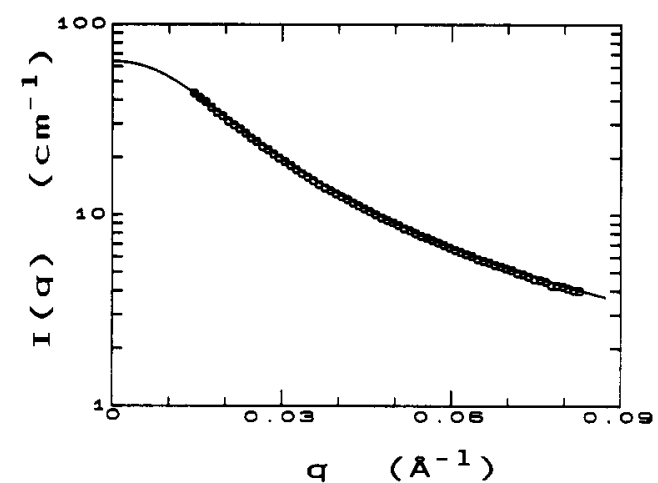

Figure 2. Scattered intensity measured for the blend at $323 \mathrm{~K}$ along with the best fit of eq 2 .

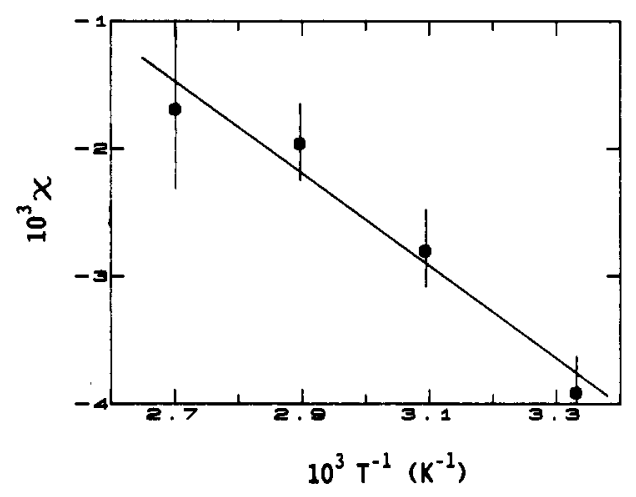

Figure 3. Temperature dependence of the interaction parameter determined from fitting eq 2 to the measured SANS intensities. The vertical bars reflect the estimated error arising from the various uncertainties underlying the application of eq 2 . The solid line corresponds to $\chi=8.3 \times 10^{-3}-3.6 / T$. The stability of the polymers at the highest temperature is problematical. Omission of the highest temperature datum gives $\chi=1.1 \times 10^{-2}$ $-4.1 / T$.

the respective pure components $\left(=6.0 \AA^{20}\right.$ and $6.6 \AA^{26}$ for PVE and PIP, respectively) could be employed; this changes the value obtained for $x$ by less than $10 \%$. The scattering curves conformed well to eq 2 through $370 \mathrm{~K}$. Representative results are shown in Figures 1 and 2. At higher temperatures significant deviations at small angles were observed, probably due to degradation of the polydienes and bubble formation from the release of dissolved air.

The values of $\chi$ determined for the blend at $300 \leq T \leq$ $370 \mathrm{~K}$ (eq 2) are displayed in Figure 3. Over all measurement temperatures the interaction parameter is less than zero, a novel circumstance for a polymer blend whose components interact only via dispersive forces. The absolute value of $\chi$ decreases with increasing temperature. This implies a lower critical solution temperature (lcst) but at temperatures well beyond the limits of the polymers' chemical stability.

As originally conceived ${ }^{27} \chi$ is proportional to the interaction enthalpy normalized by thermal energy:

$$
\chi \sim H^{\bullet} / R T
$$

Over the limited range of experimental measurements, $\chi$ values are usually found to exhibit a linear dependence on inverse temperature, but with a nonzero extrapolated value at infinite temperature. ${ }^{19,20,28-35}$ Indeed, the data in Figure 3 can be described by

$$
\chi=8.31 \times 10^{-3}-(3.6 / T)
$$

from which it is tempting to infer that the interaction parameter is composed of a temperature-dependent enthalpic part, along with a temperature-independent entropic contribution. ${ }^{36}$ To the extent such a decomposition is valid, the negative interaction parameter in the PVE/ PIP blend can be ascribed to the enthalpic component of $\chi$. The local entropic contribution is positive and diminishes the miscibility. From eq 10 it can be estimated that the heat of mixing will be negated at roughly $440 \mathrm{~K}$, which defines the blend lcst for components of infinite molecular weight.

The appropriateness of the random-phase approximation theory (eq 2) may be questioned. A more sophisticated treatment of polymer structure and thermodynamics, which includes off-lattice effects and concentrations fluctuations, has been developed. ${ }^{37,38}$ This theory demonstrates the importance of differences in local structure of the components. Structural asymmetry, due, for example, to different chain flexibilities, can increase the noncombinatorial entropy by providing increased "packing opportunities" for the chain units. This is predicted to potentially result in a negative interaction parameter even in athermal mixtures $\left(H^{e}=0\right) .{ }^{39}$ However, such a mechanism would be associated with the temperature-independent term in eq 10 and hence is probably not the cause of the negative $\chi$ in the PVE/PIP mixture.

It is not obvious that scattering from polymeric melts, which are liquids containing free volume, should be describable by eq 2 , derived using the assumption of incompressibility. ${ }^{40}$ It follows that any molecular interpretation of results arising from the application of eq 2 is problematical. Nevertheless, we will speculate on the possible origin of a negative mixing enthalpy in a van der Waals mixture.

The excess enthalpy of a van der Waals mixture depends on the energy accompanying the replacement of dispersion forces between like contacts with ones between unlike units:

$$
H^{\mathrm{e}} \sim\left(\epsilon_{11}+\epsilon_{22}\right) / 2-\epsilon_{12}
$$

The van der Waals bond energy can be described by a series whose leading term is the London formula ${ }^{41}$

$$
\epsilon_{12} \sim\left(\alpha_{1} \alpha_{2} / r^{6}\right)+\ldots
$$

where $\alpha_{i}$ is the polarizability of the $i$ th chain unit separated by $r$ from the $j$ th unit. The notion that van der Waals blends must exhibit endothermic heats of mixing arises from a disregard of any dependence of $r$ on composition. Thus, consideration of only the numerator in eq 12 in 
combination with eq 11 gives

$$
H^{e} \sim \frac{\alpha_{1}^{2}+\alpha_{2}^{2}}{2}-\alpha_{1} \alpha_{2} \geq 0
$$

This approach underlies the Lorentz-Berthelot combining rule for van der Waals interactions ${ }^{14}$

$$
\epsilon_{12}=\left|\left(\epsilon_{1} \epsilon_{2}\right)^{1 / 2}\right| \geq 0
$$

which similarly predicts positive mixing enthalpies. Positive deviations $\left(\epsilon_{12}>\left|\left(\epsilon_{1} \epsilon_{2}\right)^{1 / 2}\right|\right)$ from calculations based on eq 14 have been observed and attributed to equation of state effects. ${ }^{42}$

An origin for a negative excess enthalpy in PVE/PIP blends is apparent from inspection of eq 12. As has been discussed in consideration of the solubility of plasticizers in polymers, ${ }^{43}$ the strong distance dependence of the interaction energy can result in stronger dispersion forces between dissimilar units than for the like contacts. In fact, the negative mixing enthalpy of many polymer/ solvent solutions has been ascribed to the enhancement in contact energies caused by closer approach of the solvent molecules. ${ }^{44}$ It is proposed that the negative $\chi$ apparent herein for PVE/PIP blends may result from the enhancement of local dispersion forces engendered by subtle alterations of the short-range structure upon blending. Note that given the strong distance dependence of the van der Waals forces (eq 12) a net negative $\chi$ is not inconsistent with the absence of measurable densification upon mixing of PVE and PIP. ${ }^{11}$ This circumstance requires that $\alpha_{\text {PVE }}$ and $\alpha_{\text {PIP }}$ be essentially equal, a fact inferable from these polymers' solubility parameters. ${ }^{45}$

The presence of 1,4-butadiene units in the PVE severely reduces miscibility with PIP, with such blends exhibiting lower critical solution temperatures at observable temperatures. ${ }^{10,11}$ Recently it was found that in blends of 1,4-polyisoprene with polybutadiene containing $9 \% 1,2-$ units, while $\chi$ is always positive $\left(\geq 10^{-3}\right)$, it has an inverse temperature dependence. ${ }^{34}$ This suggests that a similar enthalpic effect as seen herein may be operative. Isotopic blends of medium vinyl polybutadiene ( $~ 50 \%$ 1,2-units) have been determined to have negative interaction parameters; 33 however, the temperature-dependent part of $\chi$ was positive, indicating an entropic origin for the negative interaction parameter.

\section{Summary}

PVE and PIP form simple van der Waals mixtures without any specific interactions. Nevertheless, as shown herein, their miscibility extends to infinite molecular weight of the components due to a negative interaction parameter. From the temperature dependence of $\chi$ it is suggested that the sign of the noncombinatorial mixing free energy may have an enthalpic origin. A mechanism for this has been suggested.

Acknowledgment. We express our appreciation to C. A. Trask (Allied-Signal Co.) for synthesizing the PVE and to N. Balsara (Exxon Chemical Co.), J. Barker, and C. J. Glinka (NIST) for experimental assistance. We gratefully acknowledge insightful comments by A. Y. Coran (Monsanto Chemical Co.). This work was supported by the Office of Naval Research, with D.W.T. as a National Research Council postdoctoral associate.

\section{References and Notes}

(1) Bates, F.S.; Koehler, W. C.; Wignall, G. D.; Fetters, L. J. Mater. Res. Sec. Symp. Proc. 1987, 159.
(2) Yang, H.; O'Reilly, J. M. Mater. Res. Soc. Symp. Proc. 1987, 129.

(3) Bates, F. S.; Dierker, S. B.; Wignall, G. D. Macromolecules 1986, 19, 1938.

(4) Ito, H.; Russell, T. P.; Wignall, G. D. Macromolecules 1987, $20,2214$.

(5) Bartenev, G. M.; Kongarov, G.S. Rubber Chem. Technol. 1963, 36,668 .

(6) Miller, J. B.; McGrath, K. J.; Roland, C. M.; Trask, C. A.; Garroway, A. N. Macromolecules 1990, 23, 4543.

(7) Roland, C. M. Macromolecules 1987, 20, 2557.

(8) Trask, C. A.; Roland, C. M. Macromolecules 1989, 22, 256.

(9) Roland, C. M. J. Polym. Sci., Polym. Phys. Ed. 1988, 26, 839.

(10) Roland, C. M.; Trask, C. A. Rubber Chem. Technol. 1989, 62, 896.

(11) Trask, C. A.; Roland, C. M. Polym. Commun. 1988, 29, 332.

(12) Zemel, I. S.; Roland, C. M. Polymer 1992, in press.

(13) Zemel, I. S.; Roland, C. M. Polymer 1992, submitted for publication.

(14) Hildebrand, J. H.; Scott, R. L. Regular Solutions; PrenticeHall: Englewood Cliffs, NJ, 1962.

(15) Lewis, G. N.; Randal, M.; Pitzer, K. S.; Brewer, L. Thermodynamics; McGraw-Hill, New York, 1961; Chapter 21.

(16) Paul, D. R., Newman, S., Eds.; Polymer Blends; Academic Press: New York, 1978.

(17) Patterson, D. Polym. Eng. Sci. 1982, 22, 64.

(18) Flory, P. J. Discuss. Faraday Soc. 1970, 49, 7.

(19) Roland, C. M.; Trask, C. A. Polym. Bull. 1988, 20, 471.

(20) Bates, F. S.; Fetters, L. J.; Wignall, G. D. Macromolecules $1988,21,1086$.

(21) Morton, M.; Fetters, L. J. Rubber Chem. Technol. 1975, 48, 359.

(22) Halasa, A. F.; Lohr, D. F.; Hall, J. E. J. Polym. Sci., Polym. Chem. Ed. 1981, 19, 1357.

(23) Glinka, C. J.; Rowe, J. M.; LaRock, J. G. J. Appl. Crystallogr. $1986,19,427$

(24) de Gennes, P.-G. Scaling Concepts in Polymer Physics; Cornell University Press: Ithaca, NY, 1979.

(25) Hadzlioannou, G.; Gilmer, J.; Stein, R. S. Polym. Bull. 1983, 9,563 .

(26) Mays, J.; Hadjichristidis, N.; Fetters, L. J. Macromolecules $1984,17,2723$.

(27) Flory, P. J. Principles of Polymer Chemistry; Cornell University Press: Ithaca, NY, 1953; Chapter 12.

(28) Maconnachie, A.; Kambour, R. P.; White, D. M.; Rostami, S.; Walsh, D. J. Macromolecules 1984, 17, 2645.

(29) Shibayama, M.; Yang, H.; Stein, R. S.; Han, C. C. Macromolecules 1985, 18, 2179.

(30) Bates, F. S.; Dierker, S. B.; Wignall, G. D. Macromolecules $1986,19,1938$.

(31) Tomlins, P. E.; Higgins, J. S. Macromolecules 1988, $21,425$.

(32) Han, C. C.; Bauer, B. J.; Clark, J. C.; Muroga, Y.; Matsushita, Y.; Okada, M.; Tran-cong, Q.; Chang, T.; Sanchez, I. C. Polymer $1988,29,2002$.

(33) Sakurai, S.; Hasegawa, H.; Hashimoto, T.; Hargis, I. G.; Aggarwas, S. L.; Han, C. C. Macromolecules 1990, 23, 451.

(34) Jinnai, H.; Hasegawa, H.; Hashimoto, T.; Han, C. C. Macromolecules 1991, 24, 282.

(35) Hasegawa, H.; Sakurai, S.; Takenaka, M.; Hashimoto, T.; Han, C. C. Macromolecules 1991, 24, 1813.

(36) Flory, P. J.; Krigbaum, W. R. Annu. Rev. Phys. Chem. 1951, 2,383

(37) Curro, J. G.; Schweizer, K. S. J. Chem. Phys. 1988, 88, 7242.

(38) Schweizer, K. S.; Curro, J. G. J. Chem. Phys. 1989, 91, 5059.

(39) Curro, J. G.; Schweizer, K. S. Macromolecules 1990, 23, 1402.

(40) Dudowica, J.; Freed, K. L. Macromolecules 1990, 23, 1519.

(41) Hirschfelder, J. O.; Curtiss, C. F.;Bird, R. B. Molecular Theory of Gases and Liquids; Wiley: New York, 1967; Chapter 13.

(42) Abe, A.; Flory, P. J. J. Am. Chem. Soc. 1966, 88, 2887.

(43) Coran, A. Y. Personal communication. See also: Coran, A. Y.; Anagnostopoulos, C. E. J. Polym. Sci. 1962, 57, 1962.

(44) Sanchez, I. C.; Lacombe, R. H. Macromolecules 1978, 11, 1145. (45) Cohen, R. E.; Wilfong, D. E. Macromolecules 1982, 15, 370.

Registry No. PVE, 9003-17-2; PIP (homopolymer), 900331-0; neutron, 12586-31-1. 